\title{
El Sistema Administrativo de Gestión de Recursos Humanos y la Ley del Servicio Civil en la Universidad Nacional del Centro del Perú - 2018 - 2019
}

\author{
The Administrative Human Resources Management System and the Civil \\ Service Law at the National University of the Center of Peru - 2018 - 2019
}

\author{
aRey Navarro, W.; ' Morales Carrión, C. \\ Facultad de Administración de Empresas / Universidad Nacional del Centro del Perú \\ E-mail:wrey@uncp.edu.pe
}

\section{Resumen}

El presente trabajo de investigación, está referido al estudio de la implementación del Sistema de Gestión de Recursos Humanos en la Universidad Nacional del Centro del Perú, establecido por la Ley del Servicio Civil, $\mathrm{N}^{\circ}$ 30057, y su Reglamento aprobado mediante el D.S. 040-2014-PCM. Habiendo transcurrido más de cuatro años de vigencia, se examina las causas del por qué la oficina de recursos humanos, no ha realizado ninguna gestión que le permita implementar este dispositivo legal, cuya efectividad permitirá mejorar las estrategias, políticas, procedimientos, relaciones humanas y clima organizacional de la gestión administrativa de los recursos humanos de la UNCP, lo que permitirá brindar un servicio de calidad.

Se realizó una encuesta a través de la aplicación de un cuestionario, a la muestra predeterminada del personal administrativo, 95 trabajadores, entre nombrados y contratados del D. L. 276 y de la Ley $\mathrm{N}^{\circ} 1057$, determinándose que muy pocos conocen la Ley del Servicio Civil, menos sobre el Sistema Administrativo de Recursos Humanos, pese a que se cumplen más de cuatro años de su puesta en vigencia y, se está a punto de cumplir el plazo, en el 2019, para realizar el tránsito a este nuevo régimen laboral. Al final, se presentan las conclusiones y recomendaciones que permitirán contar con un sistema de recursos humanos eficiente, que contribuya a elevar la calidad del servicio que se brinda a la comunidad universitaria.

Palabras clave: sistema administrativo, recursos humanos, servicio civil, clima organizacional, régimen laboral

\begin{abstract}
This research work refers to a study of the implementation of the Human Resources Management System at the National University of the Center of Peru, established by the Civil Service Law, No. 30057, and its regulations approved by the S.D. 040-2014-PCM. After more than four years of its validity, we examine the reasons why the Human Resources Office has not carried out any action that allows it to implement this legal device, whose effectiveness will allow to improve strategies, policies, procedures, human relations and organizational climate of the administrative management of human resources of the UNCP, which will allow to provide a quality service.

A survey was carried out through the application of a questionnaire to the predetermined sample of administrative personnel, 95 workers between appointed and contracted of DL 276, of Law No. 1057, determining that very few are aware of the Civil Service Law, let alone the Administrative Human Resources System, despite the fact that more than four years have passed since its entry into force and the deadline for transitioning to this new labor regime is about to be met in 2019. At the end we present our conclusions and important recommendations that will allow us to have an efficient Human Resources System, which will contribute to raising the quality of the service provided to the university community.
\end{abstract}

Keywords: administrative system, human resources, civil service, organizational climate, labor regime 


\section{Introducción}

Hablar del Sistema Administrativo de Gestión de Recursos Humanos en la administración pública, es remontarnos a 1937, cuando se crea el Sistema de Escalafón y Pensiones, posteriormente denominada Dirección General del Servicio Civil, adscrito al Ministerio de Justicia y Culto; ya en 1950, se da la Ley del Estatuto y del servicio civil y su Reglamento, que definió de manera clara la carrera administrativa en el Perú.

En materia remunerativa, entre 1969 y 1973, se estableció criterios uniformes para fijar las remuneraciones en todo el sector público; en 1978, se determinó los componentes de la remuneración y la estructura remunerativa dividida en 8 grados; en 1979, con la aprobación de la Constitución Política del Estado, tiene rango constitucional la carrera administrativa de los trabajadores y funcionarios de la administración pública; en 1984, se dicta la Ley de Bases de la Carrera Administrativa y Remuneraciones del Sector Público, dictaminando disposiciones sobre remuneraciones y pensiones de la administración pública, vigente hasta la fecha.

Después de muchos debates entre el Poder Legislativo y Poder Ejecutivo, en julio del 2013, se promulgó la Ley del Servicio Civil - Ley 30057, cuya finalidad es establecer un régimen único y exclusivo para las personas que prestan servicios en las entidades públicas del Estado; así como, para aquellas personas encargadas de su gestión. Su finalidad es que las entidades públicas del Estado alcancen mayores niveles de eficacia y eficiencia en el servicio civil, como también promover el desarrollo de las personas que lo integran; en junio del 2014, se promulga su Reglamento mediante el D.S. $\mathrm{N}^{\circ}$. 0402014- PCM.

El servicio civil se basa en el mérito, igualdad de oportunidades y el aseguramiento de la calidad de los servicios del Estado, en beneficio de la ciudadanía; el mérito busca que los puestos del servicio civil, sean ocupados por las personas idóneas, en virtud de sus habilidades, aptitudes y capacidades; siendo el fundamento en los procesos de selección, progresión en la carrera y evaluación de desempeño; de la misma manera, en la gestión de la capacitación, gestión del rendimiento y la permanencia en el servicio civil y, la mejora continua en los proceso de cada entidad, principalmente en la obtención de los resultados y metas programadas.

El objetivo fue: Determinar por qué la oficina de recursos humanos no define la planificación de políticas de recursos humanos que señala la Ley del Servicio Civil en la Universidad Nacional del Centro del Perú. La justificación está referida, principalmente, por qué los actores principales de la planificación de recursos Humanos en la universidad, como son: La Oficina General de Personal, Oficina de Control y Desarrollo de Personal, la Oficina de Remuneraciones y la Oficina de Escalafón Universitario. Pocos conocen sobre esta norma legal. También se debe responder de por qué hace más de dos años, no se ha realizado ningún seminario, curso y fórum, que permita conoce las ventajas de su implementación.

En la parte final del artículo de investigación, se plantea las conclusiones y recomendaciones, producto del trabajo de campo realizado y analizado. No ha sido fácil conseguir que los funcionarios desarrollen las encuestas, tampoco el personal administrativo, por ser receloso en brindar opiniones, pero se ha logrado un porcentaje muy importante.

\section{Métodos y materiales}

La metodología empleada, es el método científico; es un trabajo de investigación básica, no experimental y descriptiva simple. La población, está conformada por todos los trabajadores, tanto nombrados como contratados de la Universidad Nacional del Centro del Perú del D.L. N $\mathrm{N}^{\mathrm{o}} 276$ y CAS, y; la muestra, está constituida por 95 personas, que representan el $20 \%$ de cada uno de los trabajadores nombrados y contratados de los regímenes de trabajo, siendo la muestra no probabilística.

La investigación fue básica no experimental, del tipo descriptivo simple, del modelo:

\section{$\mathrm{M}-\ldots-\ldots$}

Donde “M” es la muestra donde se realiza el estudio; es decir, los trabajadores administrativos nombrados y contratados de la Universidad Nacional del Centro del Perú. Para el estudio, "O” indica la información relevante y de interés obtenida en relación con la variable en el Sistema Administrativo de Gestión de Recursos Humanos en la administración pública.

La población estudiada, fue el personal administrativo nombrado y contratado de los regímenes laborales del D.L. N ${ }^{\circ} 276$ y del Contrato Administrativo de Servicios - CAS, que labora en la Universidad Nacional del Centro del Perú.

\section{Técnicas e instrumentos de recolección de datos}

En la presente investigación, se utilizó como técnica la encuesta, por ser un procedimiento considerado clásico en las ciencias sociales para la obtención y registro de datos. Para recabar la información, se aplicó el el cuestionario, que es un formato redactado en forma de interrogatorio para obtener información acerca de las variables que se investigan, sus dimensiones y reactivos considerados.

La fiabilidad, confiabilidad, consistencia y credibilidad de la investigación se logró a través del análisis de la información, lo cual permitió internalizar las bases teóricas, el cuerpo de ideas y la realidad. 
La validez del instrumento de recolección de datos, se realizó a través de la validez de contenido; es decir, se determinó hasta donde los ítems, que contiene el instrumento, fueron representativos de lo que se desea medir.

Para el procesamiento y validación de las variables, se utilizó el programa informático Excel y las correspondientes a inferencia estadística, con el fin de tener elementos para aplicar el método analítico. Se elaboraron y analizaron las tablas y los gráficos, que son formas visibles de presentar los datos.

\section{Resultados}

De acuerdo a lo planteado, fue necesario saber si los trabajadores administrativos conocen la Ley del Servicio Civil; de los 95 trabajadores encuestados, solo 35 trabajadores conocen algo de esta norma legal, que significa el $36,84 \%$, y 60 trabajadores no lo conocen, significando el $63,16 \%$.

Sobre la implementación de políticas referidas a recursos humanos, lo cual es importante para lograr un servicio de calidad a los usuarios de la UNCP, se encontró que de las 95 personas encuestadas, solo 35 trabajadores, el 36,84\%, conocen sobre algunas políticas que hayan sido implementadas por la oficina de personal y; 60 entrevistados, el 63,16\%, lo desconocen.

Identificar requerimientos futuros de personal, implica diseñar estrategias, políticas y procedimientos, así como tener planes para el personal referidos a la planificación del éxito, considerar al talento humano como capital y fomentar el liderazgo con que cuenta la universidad; es por ello, que muy pocos encuestados conocen estos temas. Solo 29 encuestados, el 30,53 \%, saben y; 65 , que representa al 69,47\% no saben sobre este punto.

La gestión de la incorporación, es un indicativo muy preciso de la gestión del empleo, referido a la selección de personal, solo 32 encuestados, el 32,68 \% conocen; pero, 63 encuestados, es decir el $66.32 \%$, no conocen. Referido a la vinculación, solo 25 encuestados, el 25,26 $\%$ lo conocen y; 70 o el 73,68 \% no saben nada; sobre la inducción, 35 encuestadas, que son el 36,84\% conocen algo y 60 encuestadas, el 63,16\% no saben nada.

Sobre administración de personas, esta dimensión tiene como 5 indicadores: Administración de legajos, solo 30 encuestados, el 31,58 \% conocen o saben algo, y; 65 encuestados, que significa el 68,42\%, no saben.

Sobre el control de asistencia, 29 encuestados, o el $28,26 \%$ conocen y; 66 encuestados, que significa el $71,74 \%$ no saben nada. Sobre desplazamiento, de 40 encuestados, es decir el 42,11\% saben algo y; 55 encuestados, lo que significa el 57,89\% no conocen nada. Aplicación del procedimiento disciplinario, 36 encuestados, que son el 37,89\% conocen este proceso y; 59 encuestados, el 62,11\% restante no saben nada. Sobre el proceso de desvinculación, solo 30 personas conocen, lo que representa el 31,58 \% y; 65 encuestados, o el 68,42 $\%$ no saben nada.

El proceso de relaciones humanas y sociales, comprende las relaciones individuales y colectivas, nos muestra que solo 25 encuestados, el 26,32 \% conocen y; 70 encuestados, que significa el $73,68 \%$ poco o nada saben sobre este tema.

\section{Discusión}

Siendo el objetivo general el determinar por qué la oficina de recursos humanos, no ha iniciado el proceso de implementación del Sistema Administrativo de Gestión de Recursos Humanos establecidos por la Ley del Servicio Civil, en la Universidad Nacional del Centro del Perú.

El Objeto de la Ley, es establecer un régimen único y exclusivo para las personas que prestan servicios en las entidades públicas del Estado; así como, para aquellas personas que están encargadas de su gestión, del ejercicio de sus potestades y de la prestación de servicios a cargo de estas. Así mismo, la finalidad de la presente Ley es que, las entidades públicas del Estado, alcancen mayores niveles de eficacia y eficiencia y presten, efectivamente, servicios de calidad a través de un mejor servicio civil; como también, promover el desarrollo de las personas que lo integran, pese a que estos aspectos son muy importantes para el desarrollo personal, pero a muchos no les interesa conocer esta norma legal.

La implementación de un nuevo régimen de trabajo para el servidor público, donde están inmersos el personal administrativo de la Universidad Nacional del Centro del Perú; de igual manera, es necesaria que esta norma legal sea analizada, pero se ha confirmado la poca importancia que se le ha dado, ya que en la actualidad, la oficina de personal solo está aplicando toda la normatividad de la Ley $\mathrm{N}^{\circ}$ 276, Ley de Bases de la Carrera Administrativa y la Ley Contrataciones Administrativas de Servicios CAS.

El personal encuestado, que su mayoría son personas con cargos directivos con muchos años de trabajo en la Universidad, no han leído ni han analizado la Ley del Servicio Civil. Por eso, se hace muy imperioso que la oficina de recursos humanos haga la difusión y análisis correspondiente, esto es muy importante, por que es una forma de lograr una participación activa de los trabajadores administrativos con muchos años de trabajo, para evitar en lo futuro una resistencia al cambio.

Las charlas, coloquios, seminarios y talleres permitirán hacer más transversal esta normatividad. El sistema administrativo, tiene por finalidad regular la utilización de los recursos en las entidades de la administración pú- 
blica, promoviendo la eficacia y la eficiencia en su uso. Por ello, el Sistema Administrativo de Gestión de Recursos Humanos, que tiene como ente rector a Servir, tiene como finalidad contribuir a la mejora continua de la administración pública, a través del fortalecimiento del servicio civil, este organismo técnico especializado adscrito a la Presidencia del Consejo de Ministros, tiene la atribución: normativa, supervisora, sancionadora, interventora y de resolución de controversias.

De acuerdo al primer objetivo específico, que fue determinado por las encuestas realizadas, se pudo comprobar que la oficina de personal no ha implementado este subsistema de la planificación de políticas de recursos humanos en la UNCP, por que se está esperando la aprobación del Estatuto de la universidad, la misma que ha sido elaborada en base a la Ley Universitaria $N^{\circ} 30220$.

Con referencia al segundo objetivo específico, el mismo que es determinar por qué la oficina de recursos humanos no determina la gestión del empleo que estipula la Ley del Servicio Civil en la Universidad Nacional del Centro del Perú. No se ha implementado, porque se está a la espera de los documentos de gestión que deben ser elaborados por la Oficina de Racionalización y Métodos, estando en proceso de elaboración el Reglamento de Organización y Funciones ROF, lo mismo ocurre cuando se analizó los otros dos objetivos específicos.

\section{Conclusiones}

- Apesar del propósito de la Ley de Servicio Civil para establecer un régimen único y exclusivo para las personas que prestan servicios en entidades públicas del Estado, la mayoría de los servidores entrevistados desconocen los puntos más importantes de este dispositivo legal, como la gestión del conocimiento, nuevos perfiles y los procesos disciplinarios correspondientes.

- Asimismo, se determinó que no existe comunicación ni procedimientos administrativos de gestión en la universidad, referidos a la planificación de políticas de recursos humanos que permitan definir las políticas y directivas de la entidad, con una visión integral de los temas relacionados para lograr la eficiencia y la eficacia de la entidad,

- La gestión de relaciones humanas y sociales, es la que alcanza las relaciones establecidas entre la entidad y sus empleados en relación con las políticas y prácticas de personal, lo que aumenta la satisfacción y las tasas de compromiso de los trabajadores, incluyendo: a) Relaciones laborales individuales y colectivas, b) Seguridad y salud en el trabajo, c) Bienestar social, d) Cultura y clima organizacional, e) Comunicación interna.

- Finalmente, se determinó que la oficina encargada del procedimiento no ha realizado ningún trámite administrativo para ver la posibilidad de aplicación del Sistema Administrativo de Recursos Humanos en la
UNCP, pese a que Servir ha emitido muchos instrumentos de gestión, con la finalidad de implementar la Ley del Servicio Civil en las instituciones públicas de alcance nacional, regional y local.

\section{Referencias bibliográficas}

Ander - Egg. Ezequiel. (2003). Métodos y técnicas de investigación social II - La ciencia: Su método y la expresión del conocimiento científico. 1era. Ed. Buenos Aires: Lumen, 204 p.

Arbaiza Fermini, Lydia (2014). Administración y organización: Un enfoque contemporáneo. 1era. Ed.-Ciudad Autónoma de Buenos Aires: Cengage Learning Argentina, 299 pág.

Chiavenato, Idalberto (2011). Administración de recursos humanos- El capital humano de las organizaciones. Novena Edición- México, D.F. McGraw-Hill/Interamericana Editores, S.A. DE C.V. 421 p. 4.

Roberto Hernández Sampiere, Carlos Fernández Collado \& Pilar Baptista Lucio (2010). Metodología de la investigación. 5ta. Ed. México: McGraw-Hill, Interamericana Editores, S.A. DE C.V. 613 pág.

Constitución Política del Estado del año: 1933, 1979, 1993

Decreto Ley N ${ }^{\circ} 11377$, Estatuto y Escalafón del Servicio Civil

Decreto Legislativo No 276 , Ley de Bases de la Carrera Administrativa y de Remuneraciones del Sector Público.

D.S. $N^{\circ}$ 040-2014-PCM Reglamento de la Ley del Servicio Civil

Gareth R. Jones \& Jennifer M. George (2014). Administración contemporánea. Octava Edición, McGRAWHILL Interamericana Editores S.A. México D.F.

Ley Marco de la Modernización de la Gestión del Estado Ley N 27658, promulgada el 17 de enero del 2003.

Ley del Código de Ética de la Función Pública; Ley ${ }^{\circ}$ 27815. Ley del Código de Ética de la Función Pública se promulgó el 22 de julio del 2002,

Ley Marco del Empleo Público; Ley N² 28175, aprobada el 28 de enero del 2004.

Ley $N^{\circ}$ 30057, Ley del Servicio Civil

Universidad Nacional del Centro del Perú, Oficina General de Planificación, Oficina de Estadística (2018). Boletín Estadístico UNCP 2017, Impreso Fondo y Producción Editorial e Impresión. 382 págs.

Ventura Belén, Ena y Delgado Gonzáles, Susana (2013). Gestión de Recursos Humanos. 1era. Edición - Madrid España, Ediciones Paraninfo, S.A. 251 pág. 